\title{
New Bounds on Roller Coaster Permutations *
}

\author{
Fábio Botler ${ }^{1}$, Bruno L. Netto ${ }^{1}$ \\ ${ }^{1} \mathrm{PESC} / \mathrm{COPPE}$ - Universidade Federal do Rio de Janeiro (UFRJ) \\ Rio de Janeiro - RJ - Brazil \\ \{fbotler, brunoln\}@cos.ufrj.br
}

\begin{abstract}
A roller coaster is a permutation $\pi$ that maximizes the sum $\mathrm{t}(\pi)=$ $\sum_{\tau \in X(\pi)} \operatorname{id}(\tau)$, where $X(\pi)$ denotes the set of subsequences of $\pi$ with cardinality at least 3; and $\mathrm{id}(\tau)$ denotes the number of maximal increasing or decreasing subsequences of contiguous numbers of $\tau$. We denote by $\mathrm{t}_{\max }(n)$ the value $\mathrm{t}(\pi)$, where $\pi$ is a roller coaster of $\{1, \ldots, n\}$, for $n \geqslant 3$. Precise values of $t_{\max }(n)$ for $n \leqslant 13$ were presented in [Ahmed and Snevily 2013]. In this paper, we explore the problem of computing lower bounds for $\mathrm{t}_{\max }(n)$. More specifically, we present a cubic algorithm to compute $\mathrm{t}(\pi)$ for any given permutation $\pi$; and an Integer Linear Programming model to obtain roller coasters. As a result, we improve known lower bounds found in the literature for $n \leqslant 40$.
\end{abstract}

\section{Introduction}

Throughout the text, $[n]$ denotes the set $\{1, \ldots, n\}$. Fixed a positive integer $n$, a permutation $\pi$ of $[n]$ is an ordering $\left(\pi_{1}, \pi_{2}, \pi_{3}, \ldots, \pi_{n}\right)$ of $[n]$. We omit commas and parenthesis whenever doing so produces no ambiguity. The length of a permutation $\pi$ is the number of elements it contains, and we denote it by $|\pi|$. Let $S_{n}$ denote the set of all permutations of $[n]$, and let $|X|$ denote the cardinality of a set $X$. A subsequence $\tau$ of a permutation $\pi$, denoted by $\tau \subseteq \pi$, is a sequence obtained from $\pi$ by removing some (maybe none) of the elements of $\pi$, while keeping the order of the remaining elements.

Let $\mathrm{i}(\tau)$ (resp. $\mathrm{d}(\tau)$ ) be the number of maximal increasing (resp. decreasing) sequences of contiguous numbers in $\tau$, where a sequence of contiguous numbers consists of at least two consecutive numbers. Let $\mathrm{id}(\tau)=\mathrm{i}(\tau)+\mathrm{d}(\tau)$, and let $X(\pi)$ denote the set of every subsequence $\tau \subseteq \pi$ with at least three elements. Finally, we set $\mathrm{t}(\pi)=\sum_{\tau \in X(\pi)} \operatorname{id}(\tau)$. For illustration, we evaluate it on two permutations of $S_{4}$ :

$$
\begin{aligned}
\mathrm{t}(3412) & =\mathrm{id}(3412)+\mathrm{id}(341)+\mathrm{id}(342)+\mathrm{id}(312)+\mathrm{id}(412) \\
& =3+2+2+2+2=11 . \\
\mathrm{t}(1234) & =\mathrm{id}(1234)+\mathrm{id}(123)+\mathrm{id}(124)+\mathrm{id}(134)+\mathrm{id}(234) \\
& =1+1+1+1+1=5 .
\end{aligned}
$$

We define $\mathrm{t}_{\max }(n)$ as the maximum $\max _{\pi \in S_{n}} \mathrm{t}(\pi)$, and say that a permutation $\pi$ is a roller coaster if $\mathrm{t}(\pi)=\mathrm{t}_{\max }(n)$. In the example above, 1234 is not a roller coaster because $\mathrm{t}(1234)<\mathrm{t}(3412)$. On the other hand, it can be verified that 3412 is a roller coaster by checking that $\mathrm{t}_{\max }(4)=11$. Finally, $R C(n)$ denotes the set of roller coasters of length $n$.

*This research has been partially supported by Coordenação de Aperfeiçoamento de Pessoal de Nível Superior - Brasil - CAPES - Finance Code 001. F. Botler is supported by CNPq (423395/2018-1) and FAPERJ (211.305/2019). B. L. Netto is supported by CNPq (133347/2020-6). CNPq is the National Council for Scientific and Technological Development, and FAPERJ is the Rio de Janeiro Research Foundation. 
Roller Coaster permutations were introduced in [Ahmed and Snevily 2013], where the authors present values for $\mathrm{t}_{\max }(n)$ for $n \leqslant 13$, and a construction that provides lower bounds for $n \geqslant 14$. The definition of $t$ suggests a $\theta\left(2^{n}\right)$ algorithm to compute $t$, and one would take $O\left(2^{n} n\right.$ !) time exploring every permutation of length $n$ in order to obtain $\mathrm{t}_{\max }(n)$. In Section 2, we present an $O\left(n^{3}\right)$ algorithm to compute t; and in Section 3, an Integer Linear Programming model to find roller coasters, together with new bounds for $\mathrm{t}_{\text {max }}(n)$ for $n$ up to 40 .

\section{Fast Computation of $\mathbf{t}(\pi)$}

Let $\pi=\pi_{1} \pi_{2} \pi_{3} \ldots \pi_{n} \in S_{n}$ and let $1<j<n$. We call the triple $\pi_{j-1} \pi_{j} \pi_{j+1}$ a peak (resp. valley) if $\pi_{j}>\pi_{j-1}, \pi_{j+1}$ (resp. $\pi_{j}<\pi_{j-1}, \pi_{j+1}$ ). Given a subsequence $\tau$ of $\pi$, we denote by $\mathrm{p}(\tau)$ (resp. $\mathrm{v}(\tau)$ ) the number of peaks (resp. valleys) of $\tau$. For example, $p(42135)=0$ but $v(42135)=1$. Given $1 \leqslant i<j<k \leqslant n$, denote by $\Delta\left(\pi_{i}, \pi_{j}, \pi_{k}\right)$ the sum $\mathrm{p}\left(\pi_{i} \pi_{j} \pi_{k}\right)+\mathrm{v}\left(\pi_{i} \pi_{j} \pi_{k}\right)$. Finally, we say that $\pi_{i} \pi_{j} \pi_{k}$ is a triangle if $\Delta\left(\pi_{i}, \pi_{j}, \pi_{k}\right)=1$. Proposition 1. Let $\tau \in S_{n}$. Then $\mathrm{id}(\tau)=1+\mathrm{p}(\tau)+\mathrm{v}(\tau)$.

Proof. Let $r=\operatorname{id}(\tau)$, and let $\tau_{1}, \tau_{2}, \ldots, \tau_{r}$ be the maximal increasing and decreasing contiguous subsequences of $\tau$, in the order that they appear in $\tau$, where $\tau_{i}=\tau_{1}^{i} \cdots \tau_{s_{i}}^{i}$. Fix $i \in\{2, \ldots, r\}$. Note that $\tau_{s_{i-1}}^{i-1}=\tau_{1}^{i}$. By the maximality of $\tau_{i}$, we have that $\tau_{i}$ is increasing if and only if $\tau_{i-1}$ is decreasing. This implies that $\tau_{s_{i-1}-1}^{i-1} \tau_{1}^{i} \tau_{2}^{i}$ is either a peak or a valley. Moreover, these are the only peaks and valleys of $\tau$. Therefore, $\mathrm{p}(\tau)+\mathrm{v}(\tau)=$ $r-1=\mathrm{id}(\tau)-1$ as desired.

The next result provides an $O\left(n^{3}\right)$ algorithm for computing t.

Theorem 1. Let $\pi \in S_{n}$. Then

$$
\mathrm{t}(\pi)=|X(\pi)|+\sum_{1 \leqslant i<j<k \leqslant n} 2^{n-(k-i+1)} \Delta\left(\pi_{i}, \pi_{j}, \pi_{k}\right) .
$$

Proof. First, by Proposition 1, we have:

$$
\mathrm{t}(\pi)=\sum_{\tau \in X(\pi)} \mathrm{id}(\tau)=\sum_{\tau \in X(\pi)}(1+\mathrm{p}(\tau)+\mathrm{v}(\tau))=|X(\pi)|+\sum_{\tau \in X(\pi)}(\mathrm{p}(\tau)+\mathrm{v}(\tau)) .
$$

Now, we double count the cardinality of the following set.

$$
E=\left\{(\tau, \sigma): \tau=\tau_{1} \cdots \tau_{r} \in X(\pi), \sigma=\tau_{i} \tau_{i+1} \tau_{i+2}, 1 \leqslant i \leqslant r-2 \text { such that } \Delta(\sigma)=1\right\} .
$$

Let $d_{1}(\tau)$ (resp. $\left.d_{2}(\sigma)\right)$ denote $|\{(x, y) \in E: x=\tau\}|$ (resp. $\left.|\{(x, y) \in E: y=\sigma\}|\right)$. Clearly $\sum_{\tau \in X(\pi)} d_{1}(\tau)=|E|=\sum_{\sigma \in X(\pi),|\sigma|=3} d_{2}(\sigma)$, and $d_{1}(\tau)$ is the number of triangles of $\tau$, i.e., $d_{1}(\tau)=\mathrm{p}(\tau)+\mathrm{v}(\tau)$. Note that, for any subsequence $\sigma=\pi_{i} \pi_{j} \pi_{k}$ of $\pi$ for which $\Delta(\sigma)=1$, the pair $\left(w_{1} \sigma w_{2}, \sigma\right) \in E$, for every $w_{1} \subseteq \pi_{1} \ldots \pi_{i-1}$ and $w_{2} \subseteq \pi_{k+1} \ldots \pi_{n}$. Therefore, $d_{2}(\sigma)=2^{n-(k-i+1)}$, which is the number of permutations $w_{1} \sigma w_{2}$. Consequently, $\sum_{\sigma \in X(\pi),|\sigma|=3} d_{2}(\sigma)=\sum_{i<j<k} \Delta\left(\pi_{i}, \pi_{j}, \pi_{k}\right) d_{2}\left(\pi_{i} \pi_{j} \pi_{k}\right)=$ $\sum_{1 \leqslant i<j<k \leqslant n} 2^{n-(k-i+1)} \Delta\left(\pi_{i}, \pi_{j}, \pi_{k}\right)$. Therefore, we have:

$$
\sum_{\tau \in X(\pi)}(\mathrm{p}(\tau)+\mathrm{v}(\tau))=|E|=\sum_{1 \leqslant i<j<k \leqslant n} 2^{n-(k-i+1)} \Delta\left(\pi_{i}, \pi_{j}, \pi_{k}\right),
$$

which leads to the desired result. 
Let the value $b=k-i+1$ be the basis of the triangle $\pi_{i} \pi_{j} \pi_{k}$, and let $\Delta_{b}(\pi)$ be the number of triangles with basis $b$ in $\pi$. Equation (1) can be rewritten as $\mathrm{t}(\pi)=\sum_{b=3}^{n} 2^{n-b} \Delta_{b}(\pi)+|X(\pi)|$, which shows a single triangle with a smaller basis contributes more than a single triangle with larger basis. This supports the following conjecture, posed in [Ahmed and Snevily 2013], and also its strengthening anounced in [Adamczak 2016], that says that, for every $k \in\{0, \ldots,\lfloor n / 2\rfloor\}$, we have either $1 \leqslant \pi_{2 k+2} \leqslant n / 2$ and $n / 2 \leqslant \pi_{2 k+1} \leqslant n$ or $1 \leqslant \pi_{2 k+1} \leqslant n / 2$ and $n / 2 \leqslant \pi_{2 k} \leqslant n$.

Conjecture 1 (Ahmed-Snevily, 2013). If $\pi \in R C(n)$, then $\pi_{k+1}>\pi_{k}, \pi_{k+2}$ or $\pi_{k+1}<$ $\pi_{k}, \pi_{k+2}$, for every $k \in\{1, \ldots, n-2\}$.

\section{An Integer Linear Programming Model}

In this section we present an integer linear programming model to find roller coasters of a given size $n$. Its objective function is derived from Equation (1) and the main variable $\mathbf{x}=\left(x_{1}, x_{2}, \ldots, x_{n}\right)$ represents the permutation itself. We use auxiliary binary variables $p_{i, j, k}, v_{i, j, k}$ with $1 \leqslant i<j<k \leqslant n$, and $w_{i, j}$ with $1 \leqslant i<j \leqslant n$, where $p_{i, j, k}$ (resp. $v_{i, j, k}$ ) indicates whether $x_{i} x_{j} x_{k}$ is a peak (resp. a valley), and $w_{i, j}$ indicates whether $x_{i}>x_{j}$. A binary variable equals 1 if its property is satisfied, and 0 otherwise.

For $\left(x_{1}, \ldots, x_{n}\right)$ to be a permutation, we must have $x_{i} \neq x_{j}$, for every $i \neq j$, which is expressed by Equations (3b) and (3c). For $x_{i} x_{j} x_{k}$ to be a triangle, $x_{i} x_{j} x_{k}$ must be either a peak, for which we have $x_{j}>x_{i}$ and $x_{j}>x_{k}$, and can be expressed by equations $x_{j} \geqslant x_{i}-n\left(1-p_{i, j, k}\right)+1$ and $x_{j} \geqslant x_{k}-n\left(1-p_{i, j, k}\right)+1$; or a valley, for which we have $x_{j}<x_{i}$ and $x_{j}<x_{k}$, and can be expressed by equations $x_{j} \leqslant x_{k}+n\left(1-v_{i, j, k}\right)-1$ and $x_{j} \leqslant x_{i}+n\left(1-v_{i, j, k}\right)-1$. These constraints are denoted by $P V_{i, j, k}$ (see Equation (3d)).

Model 1. An Integer Programming Model for finding roller coasters.

$$
\begin{aligned}
& \max \mathrm{t}(\mathbf{x})=\sum_{1 \leqslant i<j<k \leqslant n} 2^{-(k-i+1)}\left(p_{i, j, k}+v_{i, j, k}\right) \\
& \text { s.t. } \quad w_{i, j}+w_{j, i}=1, \quad \forall i \neq j, \\
& x_{i} \geqslant x_{j}+n\left(w_{i, j}-1\right)+1, \quad \forall i \neq j, \\
& P V_{i, j, k}, \quad \forall i<j<k \text {. }
\end{aligned}
$$

Unfortunately, we were not able to run this model for $n \geqslant 18$. On the other hand, by using Adamczak's strengthening of Conjecture 1 as additional constraints, which are translated to $x_{i} \geqslant n / 2$ when $i$ is even, and $x_{i} \leqslant n / 2$ when $i$ is odd, we were able to obtain new permutations for $n$ up to 40 (see Table 2). These new permutations improved some of the lower bounds on $t_{\max }$ known so far (see Table 1). Note that if the strengthening of Conjecture 1 holds, then these additional constraints exclude only the solutions for which $x_{i} \leqslant n / 2$ when $i$ is even, and $x_{i} \geqslant n / 2$ when $i$ is odd, and hence do not exclude all optimal solutions, which implies that the permutations found are indeed roller coasters, and their respective values of $t$ are $t_{\max }$. Our experiments were written on Sagemath [The Sage Developers 2020] and ran with the Gurobi solver [Gurobi Optimization 2021].

\section{Conclusion and Future work}

This paper presents an alternative and fast algorithm to calculate $t$, and an integer linear programming model to find roller coasters, which provided us with new lower bounds 
for $t_{\max }$. We plan to explore Conjecture 1 in order to prove it or disprove it. While our methods have frequently supported the validity of Conjecture 1 , by excluding the additional constraints, the model may be able to find a counterexample for it.

Table 1. Permutations found using Model 1.

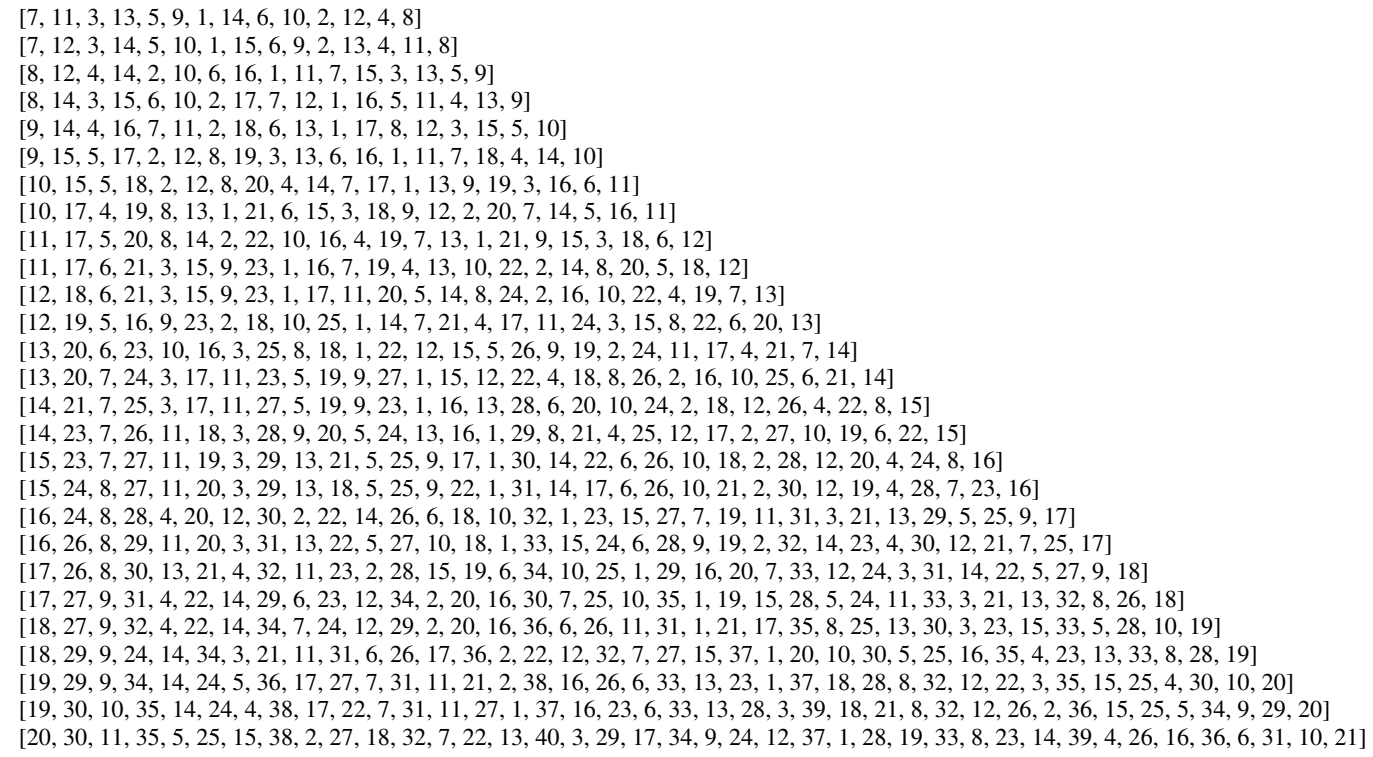

Table 2. Values of $t(n)$ obtained by 'AS', as in [Ahmed and Snevily 2013], and with 'BN', as the integer linear programming model, for $n=14, \ldots, 40$. Improved lower bounds are presented with bold text.

\begin{tabular}{|c|c|c|c|c|c|}
\hline & 14 & 15 & 16 & 17 & 18 \\
\hline AS & 81350 & 174954 & 374409 & 798471 & 1700036 \\
\hline $\mathrm{BN}$ & 81350 & 174954 & 374409 & 798783 & 1700036 \\
\hline & $-19^{-}$ & $-2 \sigma^{-}$ & $-21^{-}-$ & $-22--$ & $-23--$ \\
\hline AS & 3596124 & 7588303 & 15970785 & 33596706 & 70310126 \\
\hline $\mathrm{BN}$ & 3597020 & 7588303 & 15970785 & 33596706 & 70310126 \\
\hline & $--24--$ & $-25--$ & $-26--$ & $--27--$ & $-28-$ \\
\hline AS & 146867861 & 306492900 & 639129568 & 1327542841 & 2755084935 \\
\hline $\mathrm{BN}$ & 146867861 & 306500899 & 639198976 & 1328781760 & 2758443963 \\
\hline & $--29--$ & $--30--$ & $--\overline{3} 1^{-}--$ & $--32--$ & $--33^{--}$ \\
\hline AS & 5720021634 & 11863992638 & 24524469439 & 50593221917 & 104565405932 \\
\hline $\mathrm{BN}$ & 5720153893 & 11863992638 & 24525731250 & 50650675297 & 104569114183 \\
\hline & $--34--$ & $---35--$ & $---36--$ & $---37^{---}$ & $--38---$ \\
\hline AS & 215826275292 & 444271587981 & 914139811651 & 1881877624386 & 3872524536090 \\
\hline$\underline{\mathrm{BN}}$ & $\underline{215844113148}$ & -444587412964 & 914999923559 & 1882036116393 & 3872525917922 \\
\hline AS & 7948257224143 & 6292370258569 & & & \\
\hline $\mathrm{BN}$ & 7949294221494 & 16308000242795 & & & \\
\hline
\end{tabular}

\section{References}

Adamczak, W. (2016). A note on the structure of roller coaster permutations. arXiv preprint arXiv:1605.02415.

Ahmed, T. and Snevily, H. (2013). Some properties of roller coaster permutations. Bulletin of the Institute of Combinatorics and its Applications, 68.

Gurobi Optimization, L. (2021). Gurobi optimizer reference manual.

The Sage Developers (2020). SageMath, the Sage Mathematics Software System (Version 9.2). https: / / www. sagemath.org. 